\title{
ESTRATÉGIAS PARA REORGANIZAÇÃO DA ASSISTÊNCIA DE ENFERMAGEM À SAÚDE MATERNA FRENTE À PANDEMIA DA COVID-19
}

\author{
STRATEGIES FOR REORGANIZING MATERNAL WATER REFERENCE \\ ASSISTANCE IN FRONT OF THE COVID-19 PANDEMIC
}

Kelly Cristina Meller Sangoi ${ }^{1}$, Nadine Both da Silva ${ }^{1}$, Sandra Da Silva kinalski ${ }^{1} \&$ Vivian Lemes Lobo Bittencourt ${ }^{1}$

${ }^{1}$ Universidade Regional Integrada do Alto Uruguai e das Missóes, URI; Santo Ângelo, RS, Brasil.

\section{RESUMO}

Objetivo: realizar uma revisão integrativa da literatura sobre a reorganização e assistência nas maternidades frente à pandemia da COVID-19. Metodologia: trata-se de uma revisão integrativa de caráter exploratório e retrospectivo. A coleta de dados foi realizada de forma não sistemática no período de março a maio de 2020, na base de dados científica, PubMed, Bireme e Lilacs, e em documentos e Notas Técnicas disponibilizadas por órgãos governamentais e não governamentais. Foram utilizados os descritores "Infecçóes por coronavírus" e "Maternidade". Utilizou-se como critérios de inclusão: obedecer a temática do estudo e publicaçôes nos idiomas de português e inglês dos últimos 5 anos. Resultados: foram encontrados ao todo 213 artigos científicos, entretanto, apenas 16 atendiam aos critérios de inclusão para o alcance do objetivo proposto. Sendo que o maior número de publicaçóes incluídas foi da base de dados PubMed, além disso, a maioria dos artigos encontrados estavam na língua inglesa, e foram publicados no ano 2020. Consideraçóes finais: é de extrema importância que as maternidades se preparem para uma assistência rápida e eficaz, a fim de evitar ao máximo uma longa permanência dos pacientes em ambiente hospitalar, dando ênfase às medidas de prevenção do coranavírus. Além disso, foi possível construir conhecimento científico e uma associação de saberes populares da discente conduzidos a cientificidade, e entendimento sobre o processo de trabalho das maternidades diante a pandemia. Concluímos ainda, que está pesquisa pode colaborar com as práticas e condutas das maternidades e com a assistência de enfermagem materna durante a pandemia pelo novo coronavírus.

Descritores: Infecçóes por coronavírus; Maternidades; Cuidados de Enfermagem.

\section{ABSTRACT}

Objective: to carry out an integrative literature review on the reorganization and assistance in maternity hospitals in the face of the COVID-19 pandemic. Methodology: this is an exploratory 
and retrospective integrative review. Data collection was carried out in a non-systematic manner from March to May 2020, in the scientific database, PubMed, Bireme and Lilacs, and in documents and Technical Notes made available by governmental and non-governmental bodies. The descriptors "Coronavirus infections" and "Maternity" were used. The following inclusion criteria were used: to obey the theme of the study and publications in the languages of Portuguese and English of the last 5 years. Results: a total of 213 scientific articles were found, however, only 16 met the inclusion criteria to achieve the proposed objective. Since the largest number of publications included was from the PubMed database, moreover, most of the articles found were in the English language, and were published in the year 2020. Final considerations: it is of utmost importance that the maternities prepare for a quick and effective assistance, in order to avoid as much as possible a long stay of patients in the hospital environment, emphasizing the measures of prevention of the coranavirus. In addition, it was possible to build scientific knowledge and an association of popular knowledge of the student led to scientificity, and understanding about the work process of maternity hospitals in the face of the pandemic. We also concluded that this research can collaborate with maternity practices and conduct and with maternal nursing assistance during the pandemic due to the new coronavirus.

Descriptors: Coronavirus Infections; Maternities; Nursing care.

\section{INTRODUÇÁO}

$\mathrm{O}$ ano de 2020 foi marcado pela identificação de um novo coronavírus (SARS Cov- 2) causador da doença COVID-19 (Corona Virus Disease 19). Desde então o vírus se espalhou por várias partes do mundo e atingiu mais de 70 países. A Organização Mundial de Saúde (OMS) registrou até o dia 29 de junho de 2020, aproximadamente 10 milhóes de casos confirmados de COVID-19 com mais de 507 mil óbitos no mundo todo, sendo que 1,36 milhóes de casos confirmados e mais de 58.000 óbitos são no Brasil, onde, 75\% dos óbitos estão relacionados a algum fator de risco, como idade superior a 60 anos e doenças crônicas, como, cardiopatias, diabetes, problemas respiratórios, neurológicos e renais ${ }^{1}$.

O novo coronavírus é altamente contagioso, sua transmissão acontece principalmente de forma direta, de pessoa para pessoa, através de gotículas de saliva, tosse e espirro, ou de forma indireta por meio de contato com superfícies e objetos contaminados. A COVID-19 causa infecção respiratória, com sintomas de febre, tosse, dispneia e fadiga ${ }^{2}$.

Para diminuir e evitar novos casos, o mundo todo vem adotando medidas para prevenção da transmissão do vírus, como por exemplo o isolamento social e a conscientização de hábitos principalmente os de higiene. As informaçóes acerca da COVID-19 na gestação ainda são limitadas, estudos estão sendo realizados para maiores esclarecimentos. Até o momento não se tem registros de transmissão vertical e 
gestantes não estão no grupo de risco, todavia, devem redobrar os cuidados para evitar a contaminação pelo vírus, pois como qualquer outra doença grave pode prejudicar a saúde fetal, incluindo parto prematuro ${ }^{3}$.

O enfermeiro que atua na maternidade tem grande importância na gestáo dos cuidados de enfermagem, pois desta forma contribui para qualificar a assistência prestada. Além disso, é um mediador para novas implementaçóes de estratégias de atenção nas maternidades, baseando-se em boas práticas com evidências científicas, na humanização e no protagonismo da mulher. Portanto, é necessário que o enfermeiro se empodere cada vez mais para assumir o seu papel como gestor do cuidado obstétrico ${ }^{4}$.

Devido ao isolamento social, as maternidades estão restringindo a presença de doulas, acompanhantes e visitas, e para promover a humanização e diminuir o sentimento de solidão estão estimulando a participação virtual das famílias ${ }^{3}$.

Diante do exposto, os enfermeiros gestores das maternidades devem preparar e orientar as suas equipes para prevenir a transmissão do vírus, seguindo as orientaçôes dos órgáos governamentais, como por exemplo a precaução de contato para todas as parturientes. Se faz importante também que este profissional oriente a gestante/puérpera acerca da restriçáo de visitas, troca de acompanhantes e ressalte a importância do uso de máscaras cirúrgicas e a higienização das mãos antes de qualquer contato com o recémnascido 5 .

As equipes assistenciais podem ser orientadas para realizar uma anamnese detalhada a fim de identificar fatores de risco associados à possível contaminação pela COVID-19. Além de restringir as visitas nas maternidades existe a possibilidade de que as equipes sejam reduzidas durante todo o processo de pré-parto, parto e pós-parto, para não aumentar a exposição dos profissionais de saúde a infecção. Os profissionais devem higienizar as mãos frequentemente e utilizar Equipamentos de Proteçáo Individual (EPIs) como, avental, luvas de procedimento, máscara cirúrgica entre outros, conforme as necessidades dos procedimentos ${ }^{6}$.

O alojamento conjunto está mantido, desde que se tenha uma distância de dois metros entre a cama da mãe e o berço do recém-nascido, e que sejam utilizadas as recomendações de prevenção e controle de infecção, ressaltando a presença apenas de um acompanhante regular o qual deve ser assintomático. Entretanto, se o alojamento conjunto for em espaços compartilhados é sugerido que seja suspenso a presença de acompanhantes a fim de reduzir aglomeração de pessoas e proteger as máes e recémnascidos internados?

Além do desafio de combater a pandemia da COVID-19, a enfermagem tem o desafio de criar novas estratégias para humanizar a sua prática profissional. No cenário obstétrico, a enfermagem pode desenvolver habilidades buscando por inovaçóes criativas 
associadas à tecnologia para conseguir lidar com a complexidade vivenciada no momento e humanizar a chegada de um novo ser $^{8}$.

Cada vez mais, as instituições de atenção à mulher buscam qualificar suas equipes, onde o enfermeiro é protagonista do cuidado, da gestão e organização de fluxos e atendimentos. Portanto, este estudo é relevante pelo fato de ter o intuito de buscar e construir entendimento sobre a assistência de enfermagem nas melhores práticas durante a pandemia da COVID-19 na assistência à mulher.

Esta pesquisa poderá colaborar com melhores práticas durante este período para outros serviços de atenção à mulher que estão em processo de organização de suas condutas durante a pandemia da COVID-19. Para tanto, desenvolveu-se um estudo de revisão integrativa com a seguinte questão norteadora: Como organizar a assistência nas maternidades em resposta à pandemia pela COVID-19? Para responder a este questionamento, objetivou-se realizar uma revisão integrativa da literatura sobre a assistência nas maternidades frente à pandemia da COVID-19.

\section{METODOLOGIA}

O presente estudo foi realizado por uma discente, sob supervisão dos docentes, na disciplina de Estágio Supervisionado IE cujo objetivos da disciplina são oportunizar ao educando a vivência de situaçóes do cotidiano profissional da gestão e da assistência de enfermagem, aprimorar e consolidar as habilidades e competências inerentes a atuação do(a) Enfermeiro(a) no exercício da atividade pré-profissional, pertencente ao 90 semestre do curso de Graduação em Enfermagem de uma universidade privada da regiáo noroeste do Estado do Rio Grande do Sul.

A presente pesquisa trata-se de uma revisão integrativa de caráter exploratório e retrospectivo. A revisão integrativa, é um método de pesquisa criteriosa, que objetiva e possibilita fornecer informaçóes mais amplas e abrangentes, ademais, busca sintetizar resultados de forma sistematizada e ordenada sobre determinados temas?

De acordo com Souza, Silva e Carvalho (2010), as revisões integrativas são ferramentas fundamentais para fundamentar as práticas de saúde com embasamento científico, uma vez que, sintetizam e abrangem pesquisas disponíveis sobre determinados assuntos, possibilitando práticas baseadas em evidências ${ }^{10}$.

O processo para realização da coleta de dados deu-se de forma não sistemática no período de março a maio de 2020 nas seguintes bases de dados: Biblioteca Nacional de Medicina dos Estados Unidos (PubMed), Biblioteca Regional da medicina (BIREME) e Literatura Latino-Americana em Ciências da Saúde (LILACS), buscando a temática “assistência nas maternidades frente à pandemia da COVID-19” através dos descritores “infecções por coronavírus" e "maternidade”. Além disso, também foram explorados 
documentos e notas técnicas sobre a temática publicados pelos órgãos governamentais e não governamentais.

Como critérios de inclusão para desenvolver a discussão da presente revisão levouse em consideração: artigos e documentos que apresentavam informaçóes referente a temática, nos idiomas de português e inglês e que foram publicados nos últimos 5 anos. Diante disso, excluíram-se artigos e documentos que náo se encaixavam no assunto a ser tratado na revisão, que não estavam disponíveis na íntegra, que não se enquadravam no recorte temporal, que náo eram redigidos nos idiomas supracitados, e que se encontravam em duplicata em outras bases de dados.

\section{RESULTADOS E DISCUSSÓES}

Foram encontrados ao todo 213 artigos científicos, entretanto apenas 16 atendiam aos critérios de inclusão para o alcance do objetivo proposto. Sendo que o maior número de publicaçóes incluídas foi da base de dados da PubMed, onde encontramos 205 publicaçóes, destas, 12 atendiam aos critérios de inclusão. Na sequência a base de dados BIREME com duas publicaçóes, porém, apenas uma atendia ao objetivo proposto. Logo na base de dados LILACS, foi encontrado apenas um documento atendendo aos critérios de inclusão. Já nos órgãos governamentais, foram encontrados três documentos no Ministério da Saúde, destes foi selecionado um, pois dois já haviam sido encontrados nas demais buscas. Nos órgãos não governamentais foram encontrados dois documentos na Sociedade Brasileira de Pediatria, destes foi selecionado apenas um, pois o outro já havia sido selecionado na base de dados BIREME.

A seguir, encontra-se a relação dos artigos selecionados e analisados: 
Tabela 1. Artigos selecionados e analisados. Santo Ângelo, RS, Brasil, 2020 (n=16)

\begin{tabular}{|c|c|c|c|c|}
\hline & TÍTULO & AUTORES & $\begin{array}{c}\text { ANO DE } \\
\text { PUBLICAÇÁO }\end{array}$ & $\begin{array}{c}\text { SÍNTESE DO } \\
\text { ARTIGO }\end{array}$ \\
\hline 1. & $\begin{array}{l}\text { Força- Tarefa de Obstetrícia } \\
\text { COVID-19, Lombardia, Itália: } \\
\text { resumo da gerência executiva e } \\
\text { breve relatório de resultado }{ }^{11} \text {. }\end{array}$ & $\begin{array}{l}\text { FERRAZZI, E. M., et } \\
\text { al. }\end{array}$ & 2020 & $\begin{array}{l}\text { Relata o resultado da gerencia } \\
\text { executiva no combate a } \\
\text { COVID-19. }\end{array}$ \\
\hline 2. & $\begin{array}{l}\text { Manejo perinatal-neonatal da } \\
\text { infecção por COVID-19 - } \\
\text { Diretrizes da Federaçáo das } \\
\text { Sociedades Obstétricas e } \\
\text { Ginecológicas da Índia (FOGSI), } \\
\text { Fórum Nacional de Neonatologia } \\
\text { da Índia (NNF) e Academia } \\
\text { Indiana de Pediatria (IAP) }{ }^{12} \text {. }\end{array}$ & CHAWLA, D., et al. & 2020 & $\begin{array}{l}\text { Fornece recomendaçóes para } \\
\text { prevençáo de transmissáo, } \\
\text { diagnóstico de infecção e } \\
\text { atendimento clínico durante o } \\
\text { trabalho de parto, ressuscitação } \\
\text { e período pós-natal. }\end{array}$ \\
\hline
\end{tabular}

3. Preparando uma unidade obstétrica no coraçáo da greve epidêmica do COVID-19: dicas rápidas de reorganização ${ }^{13}$.
CAPANNA, F., et al.

4. Maternidade proteçáo também durante a crise corona ${ }^{14}$.
MICHELS, G.; OCHMANN, U.; CRANEN, R.
2020

2020

Sugere rapidamente pontoschave de estratégias para implementar em unidades obstétricas sem demora para responder à onda que se aproxima, com base na experiência e feedback.

Recomendaçóes para a licença maternidade durante a pandemia por COVID-19.
5. Doença de Coronavírus 2019 gravidez ${ }^{15}$.
(COVID-19) Pandemia e al

DASHRAATH, P., et

al.

CHEN, D., et al.

2020

6. Consenso de especialistas para o gerenciamento de gestantes e neonatos nascidos de máes com suspeita ou confirmação de nova infecção por coronavírus (COVID-19) ${ }^{16}$.

7. Coronavírus na gravidez e no MULLINS, E., et al.

2020 parto: revisão rápida ${ }^{17}$
Compartilha uma estrutura que pode ser adotada pelas maternidades terciárias que gerenciam as gestantes no fluxo de uma pandemia, mantendo a segurança do paciente e do profissional de saúde.

Fornece diretrizes de gerenciamento clínico para o novo coronavírus (COVID-19) na gravidez.
Orienta a política de saúde e o gerenciamento de mulheres afetadas pelo COVID19 durante a gravidez. 
8. Resultados maternos e neonatais de gestantes com pneumonia por COVID-19: um estudo casocontrole ${ }^{18}$.
LI, N., et al. 2020

PENAHI, L.; AMIRI,

2020

9. Riscos da nova doença de coronavírus (COVID-19) na gravidez; uma revisão narrativa ${ }^{19}$.

\section{H.; POUY, S.}

WANG, S. S., et al.

2020

10. Experiência de manejo clínico de mulheres grávidas e recémnascidos com nova pneumonia por coronavírus no Hospital Tongji, China ${ }^{20}$.

11.

Pandemia de Coronavírus

Preocupaçóes durante a Gravidez; uma carta ao editor ${ }^{21}$.

Estudo de caso-controle para comparar características clínicas, resultados maternos e neonatais de mulheres grávidas com e sem pneumonia por COVID-19.

Revisão da literatura publicada a respeito dos riscos do coronavírus na gestaçáo.

Revisão sobre as orientações do manejo materno e fetal durante a COVID-19 e enfocou nas questóes de maior preocupaçáo das gestantes.

FAKARI, F. R.; SIMBAR, M.

2020

Principais motivos de preocupaçóes entre gestantes e dá providências para diminuir tais preocupaçôes.
12.

Desafios globais em saúde e assistência médica para enfermeiras e parteiras em todos os lugares ${ }^{22}$.

\section{CATTON, H.}

13. Prevenção e Abordagem da Sociedade Brasileira Infecçáo por COVID-19 em de Pediatria. mães e Recém-Nascidos, em Hospitais-Maternidades ${ }^{23}$.

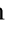

(2)

\section{Sociedade Brasileira} de Pediatria.

ao Recém-Nascido na sala de parto de máe com COVID-19 suspeita ou confirmada ${ }^{6}$.

15. Nota Técnica $n^{\circ} 10 / 2020^{7}$.
Ministério da Saúde.
16. Recomendações para Secretaria Nacional de enfrentamento ao COVID-19 Políticas para as pelo público específico de Mulheres. gestantes, lactantes e mães de bebês de até 24 meses $^{24}$.

\section{Recomendaçôes para a} assistência aos recém-nascidos filhos de mães com suspeita ou confirmação da COVID-19.

Estratégias e recomendaçôes para a atenção à saúde do recém-nascido no contexto da infecçấo pelo novo coronavírus.

Relato dos desafios enfrentados pelos enfermeiros e a sua importância para enfrentar os futuros desafios.

Disponibiliza orientações para sistematizar fluxos assistenciais práticas clínicas durante $o$ período da pandemia pelo novo coronavírus.

Orientaçóes e recomendaçóes para gestantes, lactantes e para mães de bebês de até 24 meses para o enfrentamento da COVID-19.

Fonte: Dados da Pesquisa.

A maioria dos artigos encontrados estavam na língua inglesa, portanto, se fez necessário a tradução para a língua portuguesa, e todos os artigos e documentos encontrados foram publicados no ano de 2020. 
Devemos considerar que o presente artigo por ser uma revisão integrativa, traz discussóes abrangentes acerca do tema abordado, bem como, mostra a sociedade aspectos relacionados à assistência de enfermagem nas maternidades frente a pandemia da COVID-19, e possibilita embasamento científico para as práticas assistenciais.

Após a leitura e tradução dos artigos que atendiam aos critérios de inclusão do presente estudo, optou-se a partir dos tópicos mais relevantes, pela construção de dois eixos temáticos, descritos a seguir: "Gerenciamento do fluxo de atendimento nas maternidades e assistência de enfermagem durante a pandemia da COVID-19" e "Recomendaçóes sobre prevenção e transmissão do novo coronavírus na Maternidade”.

\section{Gerenciamento do fluxo de atendimento nas maternidades e assistência de enfermagem durante a pandemia da COVID-19}

Diante da pandemia da COVID-19 instalada mundialmente, os serviços de saúde vêm adotando medidas de prevenção e controle da disseminação do novo coronavírus (SARS Cov-2), através de recomendaçóes de órgãos governamentais e orientaçóes de estudos já publicados. Com isso, o fluxo, organização e assistência das maternidades estão se adequando para prestar uma assistência mais segura para as mulheres e recém-nascidos internados, bem como, para proteger os profissionais de saúde.

Um estudo realizado com o objetivo de sugerir novas estratégias para implementar em unidades obstétricas, aponta, que é de extrema importância separar as gestantes/ puérperas com COVID-19 confirmada das demais que não possuem o vírus, criando assim uma zona para o COVID-19 dentro das maternidades ${ }^{13}$. O estudo aponta ainda, que as triagens iniciais das gestantes devem ser feitas preferencialmente fora do hospital, através de uma estrutura móvel ou por telefone, entretanto, se náo for possível desta forma, o hospital deve disponibilizar um quarto ou local isolado específico para avaliar as gestantes, antes da admissão das mesmas para a maternidade. Ressaltando que este quarto deve ter boa ventilação e deverá ser higienizado antes de depois dos atendimentos ${ }^{13}$.

Ainda, Capanna et al., 2020, disponibiliza orientaçóes para o parto e pós parto, como, cesarianas somente com indicação obstétrica, se paciente com COVID-19 confirmada a indução do parto deve ser adiada se possível, entretanto, se a paciente for sintomática, deve-se avaliar os riscos e benefícios de uma cesariana, a fim, de melhorar a função pulmonar da mulher; equipe reduzida; presença do pai somente se for assintomático; restrição da presença de doulas e/ou fotógrafos; e, a paciente deve fazer uso de máscara cirúrgica durante todo o processo de parto e puerpério ${ }^{13}$.

Já sobre a via e o momento do parto, Chen et al., 2020, afirma que não há evidências científicas comprovando qual o melhor momento e qual via de parto é mais segura, portanto, o momento ideal e a via de parto devem ser individualizados, 
baseado no bem-estar materno e fetal e na idade gestacional. A via de parto deve basearse em indicaçóes obstétricas, dando preferência ao parto vaginal sempre que possível ${ }^{16}$. Afirmação esta que corrobora o estudo de Mullins et al., 2020, que também aponta que o modo de parto deve ser determinado por indicaçáo de obstetras, e, ainda salienta a separação das mães e dos bebês que forem infectados pelo vírus da COVID-19.

Para as parturientes assintomáticas e que não tiveram contato com ninguém que apresentava sintomas respiratórios, recomenda-se o contato pele a pele e o aleitamento materno na primeira hora de vida do bebê, no entanto, parturientes sintomáticas ou que tiveram contato com pessoas com sintomas respiratórios, o contato pele a pele está suspenso, e o aleitamento materno deve ser adiado para depois que tiverem sido adotados os cuidados de higiene e medidas de prevenção de contaminaçãa ${ }^{24}$.

$\mathrm{O}$ enfermeiro gestor das maternidades, tem papel fundamental no combate a COVID-19, gerenciando, supervisionando, orientando, capacitando e apoiando as suas equipes, para que assim todos possam prestar uma assistência qualificada, eficaz e segura.

As maternidades são consideradas na maioria das vezes um ambiente de alegria e de acolhimento; com a pandemia pela COVID-19, o fluxo e a assistência estáo modificados, o que acaba deixando o ambiente menos acolhedor e humanizado, diante disso, o enfermeiro gestor é essencial para preparar as suas equipes, para que estas saibam criar estratégias para humanizar o nascimento, como por exemplo, promover o vínculo afetivo através do aleitamento materno ou pelo contato visual da mãe com o bebê, quando não for possível outras estratégias.

Corroborando as orientaçôes apontadas por Capanna et al., a Sociedade Brasileira de Pediatria, 2020, afirma que o acompanhante não pode apresentar sintomas respiratórios, não pode ter tido contato com pessoas com COVID-19 e nem estar no grupo de risco da doença, além disso, o acompanhante só é permitido em maternidades que tenham alojamento conjunto em quartos individuais/privativos, assegurando as recomendaçóes de higiene ${ }^{13 ; 23}$.

\section{Recomendaçóes sobre prevenção e transmissão do novo coronavírus na Maternidade}

Gestantes e puérperas representam um grupo vulnerável a qualquer doença infecciosa, devido a alteraçóes fisiológicas, hormonais e comprometimento do sistema imunológico. Apesar de, ainda não existir evidências científicas comprovando a transmissão vertical da COVID-19, precauçôes especiais são necessárias para diminuir o risco de transmissão do vírus, pois, a necessidade de proteger o feto aumenta o desafio de manter/controlar a saúde da mulher ${ }^{15}$.

Capanna, et al., 2020, afirma que a contenção, como, isolamento e deteç̧ão precoce é a melhor estratégia para quebrar a cadeia de transmissão do novo coronavírus, 
além disso, desta forma é possível evitar a sobrecarga dos hospitais e o colapso das infraestruturas de saúde. Diante disso, os autores citam alguns pontos a serem seguidos para prevenir a transmissão do vírus e assim evitar a sobrecarga dos hospitais e profissionais de saúde, como: realizar reunióes regulares com todos os membros da equipe das maternidades, a fim de, estudar os recursos disponíveis e tomar decisōes compartilhadas; disponibilizar um número de telefone para ligaçōes gratuitas, para as gestantes tirarem suas dúvidas antes de se deslocarem para as maternidades; e, ainda disponibilizar uma equipe de triagem móvel para atendimento e orientaçóes no domicílio das gestantes, a fim de evitar a ida desnecessária ao hospital ${ }^{13}$.

Em maternidades, cuja estrutura física náo permite o distanciamento entre os leitos de no mínimo dois metros, a permanência de acompanhante e presença de visitas deve ser suspensa durante a pandemia, a fim de prevenir a transmissão do novo coronavírus $^{23}$. Sabe-se que o nascimento é um momento único e muito esperado, mas neste momento recomenda-se que os familiares não visitem o recém-nascido e a puérpera na maternidade.

Ao prestar assistência às mulheres com suspeita ou confirmaçáo da COVID-19, os profissionais de saúde devem utilizar precauçáo padráo além dos EPIs para evitar contaminaçáo. Os EPIs devem conter máscara N95 e óculos de proteçâa ${ }^{12}$. Nem todos os pacientes apresentam sintomas da COVID-19, portanto, deve-se adotar essas medidas de precaução padráo e prevenção para todos os pacientes, independente se for ou não suspeito.

O enfermeiro gestor da maternidade deve estar constantemente orientando as suas equipes, bem como, as pacientes e seus acompanhantes, sobre as diversas formas de conter a disseminação do vírus, a fim de, conscientizar os mesmos sobre a importância das medidas de prevenção. A antecipação é fundamental, preparar as maternidades estruturalmente da forma mais eficiente, com protocolos e equipe treinada é essencial para conter a propagaçáo da COVID-19.

As recomendações contidas neste artigo relatam as mudanças de várias instituições que se empenharam para colocar em prática descobertas sobre a repercussão desta doença em gestantes e puérperas. Assim, a pandemia da COVID-19 deverá fortalecer o trabalho nas maternidades entre as equipes de saúde e os gestores.

\section{CONSIDERAÇÓES FINAIS}

A partir deste estudo, compreende-se que, é de extrema importância que as maternidades se preparem para uma assistência rápida e eficaz, a fim de evitar ao máximo uma longa permanência das pacientes em ambiente hospitalar, dando ênfase às medidas de prevenção de contaminaçáo pelo novo coronavírus. Evidencia-se ainda, que 
o profissional enfermeiro tem papel fundamental na implementação de medidas para o combate da pandemia na maternidade.

Constatamos que os estudos voltados diretamente as maternidades em tempos de pandemia pelo novo coronavírus são incipientes, devido ser uma temática recente, portanto, sugere-se novas pesquisas e maior produção de conhecimento sobre essa temática. Vale ressaltar, que, por ser uma temática prematura e que está em constante investigação, as informações presentes neste estudo podem sofrer alteraçôes.

Foi possível construir conhecimento científico e uma associação de saberes populares da discente conduzidos a cientificidade, e entendimento sobre o processo de trabalho das maternidades diante a pandemia. Concluímos ainda, que está pesquisa pode colaborar com as práticas e condutas das maternidades e com a assistência de enfermagem materna durante a pandemia pelo novo coronavírus.

\section{REFERÊNCIAS}

Brasil. Ministério da Saúde/ Secretaria de Vigilância e Saúde. Boletim Epidemiológico 08. Brasília DF. 2020. Disponível em: <https://portalarquivos.saude.gov.br/images/ pdf/2020/April/09/be-covid-08-final-2.pdf> Acesso em: 09 abr. 2020.

Brasil. Ministério da Saúde (MS). Coronavírus. Brasília DF. 2020. Disponível em: $<$ https://coronavirus.saude.gov.br/> Acesso em: 22 abr. 2020.

Osanan GC; et al. Coronavírus na gravidez: consideraçóes e recomendaçóes Sogimig. Belo Horizonte MG. Mar., 2020. Disponível em: <file://C:/Users/arthu/ Downloads/CORONAVIRUS NA GRAVIDEZ SOGIMIG . 20 DE MARC O. pdf.pdf> Acesso em: 10 abr. 2020.

Backes MTS; et al. Desafios da gestáo do cuidado de enfermagem para a qualidade da atenção obstétrica e neonatal em maternidades públicas brasileiras. Investigaçáo Qualitativa em Saúde/ CIAIQ. 2017. Disponível em: $<$ https://proceedings.ciaiq.org/ index.php/ciaiq2017/article/view/1232/1193> Acesso em: 13 abr. 2020.

\section{Schuengue N. COVID-19 em crianças: o que o enfermeiro precisa saber?}

PEBMED. 2020. Disponível em: <https://pebmed.com.br/covid-19-em-criancas-oque-o-enfermeiro-precisa-saber/> Acesso em: 16 abr. 2020.

Sociedade Brasileira de Pediatria. Recomendaçóes para Assistência ao RecémNascido na sala de parto de máe com COVID-19 suspeita ou confirmada. 2020. Disponível em: <https://www.sbp.com.br/fileadmin/user_upload/22422b-NAlertaAssist_RN_SalaParto_de_mae_com_COVID-19.pdf> Acesso em: 16 abr. 2020. 
Brasil. Ministério da Saúde (MS). Nota Técnica n 10/2020 - COCAM/CGCIVI/ DAPES/SAPS/MS. Brasília DF. 2020. Disponível em:<https://www.unasus.gov.br/ especial/covid19/pdf/97> Acesso em: 17 abr. 2020.

Preto VA; et al. Refletindo sobre as contribuições da enfermagem para a saúde global. Revista Gaúcha Enfermagem. 2015. Disponível em: <http://www.scielo.br/scielo. php?script=sci arttext\&pid=S1983-14472015000500267\&lang=pt $>$ Acesso em: 13 abr. 2020.

Ercole FF; Melo LS; Alcoforado CLGC. Revisão Integrativa versus Revisão Sistemática. Revista Mineira de Enfermagem. 2014. Disponível em: <https://cdn.publisher.gn1. link/reme.org.br/pdf/v18n1a01.pdf>. Acesso em: 07 dez. 2020.

Souza MT; Silva MD; Carvalho R. Revisão integrativa: o que é e como fazer. Revista Einstein. 2010. Disponível em: <https://www.scielo.br/pdf/eins/v8n1/pt 1679-4508eins-8-1-0102.pdf >. Acesso em: 07 dez. 2020.

Ferrazzi EM; et al. Força Tarefa de Obstetrícia COVID-19, Lombardia, Itália: resumo da gerência executiva e breve relatório de resultado. International Journal of Gynecology and Obstetrics. 2020. Disponível em: <https://obgyn.onlinelibrary.wiley. com/doi/abs/10.1002/ijgo.13162> Acesso em: 11 abr. 2020.

Chawla D; et al. Manejo perinatal-neonatal da infecção por COVID-19 - Diretrizes da Federação das Sociedades Obstétricas e Ginecológicas da Índia (FOGSI), Fórum Nacional de Neonatologia da Índia (NNF) e Academia Indiana de Pediatria (IAP). Indian Pediatrics. 2020. Disponível em: <https://www.ncbi.nlm.nih.gov/ pubmed/32238615> Acesso em: 11 abr. 2020.

Capanna F; et al. Preparando uma unidade obstétrica no coração da greve epidêmica do COVID-19: dicas rápidas de reorganização. The Journal of Maternal-Fetal \& Neonatal Medicine. 2020. Disponível em: <https://www.ncbi.nlm.nih.gov/ pubmed/32223490> Acesso em: 11 abr. 2020.

Michels G; Ochmann U; Cranen R. Maternidade, proteção também durante a crise corona. Medicine Klin Intensivmed Notfmed. 2020. Disponível em: <https://www. ncbi.nlm.nih.gov/pmc/articles/PMC7100415/> Acesso em: 11 abr. 2020.

Dashraath P; et al. Doença de Coronavírus 2019 (COVID-19) Pandemia e gravidez. American Journal of Obstetrics and Gynecology. 2020. Disponível em: < https:// www.ncbi.nlm.nih.gov/pubmed/32217113 > Acesso em: 12 abr. 2020.

Chen D; et al. Consenso de especialistas para o gerenciamento de gestantes e neonatos nascidos de mães com suspeita ou confirmação de nova infecção por coronavírus (COVID-19). International Journal of Gynecology and Obstetrics. 2020. Disponível em: <https://www.ncbi.nlm.nih.gov/pubmed/32196655> Acesso em: 11 abr. 2020. 
Mullins E; et al. Coronavírus na gravidez e no parto: revisão rápida. Ultrasound in Obstetrics and Ginecology. 2020. Disponível em: $<$ https://obgyn.onlinelibrary.wiley. com/doi/abs/10.1002/uog.22014 > Acesso em: 11 abr. 2020.

$\mathrm{Li} \mathrm{N}$; et al. Resultados maternos e neonatais de gestantes com pneumonia por COVID-19: um estudo caso-controle. Clinical Infectious Diseases. 2020. Disponível em: $<$ https://academic.oup.com/cid/article/doi/10.1093/cid/ciaa352/5813589> Acesso em: 16 abr. 2020.

Penahi L; Amiri H; Pouy S. Riscos da nova doença de coronavírus (COVID-19) na gravidez; uma revisão narrativa. Archives Academic Emergency Medicine. 2020. Disponível em: $<$ https://www.ncbi.nlm.nih.gov/pmc/articles/PMC7092922/> Acesso em: 16 abr. 2020.

Wang SS; et al. Experiência de manejo clínico de mulheres grávidas e recém-nascidos com nova pneumonia por coronavírus no Hospital Tongji, China. Current Medical Science. 2020. Disponível em: <https://www.ncbi.nlm.nih.gov/pubmed/32219626> Acesso em: 16 abr. 2020.

Fakari FR; Simbar M. Pandemia de Coronavírus e Preocupaçóes durante a Gravidez; uma carta ao editor. Archives of Academic Emergency Medicine. 2020. Disponível em: <https://www.ncbi.nlm.nih.gov/pmc/articles/PMC7075675/> Acesso em: 16 abr. 2020 .

Catton H. Desafios globais em saúde e assistência médica para enfermeiras e parteiras em todos os lugares. International Nursing Review. 2020. Disponível em: $<\underline{\text { https:// }}$ onlinelibrary.wiley.com/doi/full/10.1111/inr.12578> Acesso em: 16 abr. 2020.

Sociedade Brasileira de Pediatria. Prevençáo e Abordagem da Infecçáo por COVID-19 em mães e Recém-Nascidos, em Hospitais-Maternidades. 2020.

Disponível em: <https://pesquisa.bvsalud.org/portal/resource/pt/lis-LISBR1.1-47116> Acesso em: 11 abr. 2020.

Brasil. Ministério da Mulher, da Família e dos Direitos Humanos/ Secretaria Nacional de Políticas para as Mulheres. Recomendaçóes para enfrentamento ao COVID-19 pelo público específico de gestantes, lactantes e máes de bebês de até $\mathbf{2 4}$ meses. Brasília DF. 2020. Disponível em: <https://www.gov.br/mdh/pt-br/assuntos/ noticias/2020-2/abril/SEI_MDH1142234Ofcioassinado.pdf/view> Acesso em: 22 abr. 2020 .

Autor Correspondente: Kelly Cristina Meller Sangoi E-mail: kellysangoi@gmail.com Recebido em: 2020-07-14 